\title{
Finite-Time Bounded Synchronization of the Growing Complex Network with Nondelayed and Delayed Coupling
}

\author{
Yuhua Xu, ${ }^{1,2,3}$ Jincheng Zhang, ${ }^{4}$ Wuneng Zhou, ${ }^{5}$ and Dongbing Tong ${ }^{6}$ \\ ${ }^{1}$ School of Finance, Nanjing Audit University, Jiangsu 211815, China \\ ${ }^{2}$ Jiangsu Key Laboratory of Financial Engineering, Nanjing Audit University, Jiangsu 211815, China \\ ${ }^{3}$ Department of Mathematics and Finance, Hanjiang Normal University, Hubei 442000, China \\ ${ }^{4}$ School of Engineering Management, Nanjing Audit University, Jiangsu 211815, China \\ ${ }^{5}$ College of Information Science and Technology, Donghua University, Shanghai 201620, China \\ ${ }^{6}$ College of Electronic and Electrical Engineering, Shanghai University of Engineering Science, Shanghai 201621, China
}

Correspondence should be addressed to Yuhua Xu; yuhuaxu2004@163.com and Jincheng Zhang; zjc@nau.edu.cn

Received 17 September 2016; Accepted 13 December 2016; Published 30 January 2017

Academic Editor: Seenith Sivasundaram

Copyright (C) 2017 Yuhua Xu et al. This is an open access article distributed under the Creative Commons Attribution License, which permits unrestricted use, distribution, and reproduction in any medium, provided the original work is properly cited.

\begin{abstract}
The objective of this paper is to discuss finite-time bounded synchronization for a class of the growing complex network with nondelayed and delayed coupling. In order to realize finite-time synchronization of complex networks, a new finite-time stable theory is proposed; effective criteria are developed to realize synchronization of the growing complex dynamical network in finite time. Moreover, the error of two growing networks is bounded simultaneously in the process of finite-time synchronization. Finally, some numerical examples are provided to verify the theoretical results established in this paper.
\end{abstract}

\section{Introduction}

In recent years, as finite-time control is an important indicator of test control of complex networks, which not only has important theoretical significance, but also has important practical value in practical engineering, finite-time control of complex networks has become one of the most interesting subjects in control theory of complex networks. To get fast convergence speed, much finite-time stabilization theory is proposed to investigate finite-time control of complex networks [1-10]. For example, in [11], Shen et al. investigated finite-time synchronization control of uncertain Markov jump neural networks by input constraints. In [12], Zhang et al. discussed finite-time stabilization of time-varying nonlinear systems by state feedback control. In [13], Wang et al. discussed finite-time global synchronization of Markovian jump complex networks by partially unknown transition rates with feedback control. In [14], Liu et al. considered finite-time synchronization of Markovian switching neutral complex networks based on pinning controller. In [15], Shi studied finite-time control of linear systems with timevarying sampling, and so on. It is noticed that most of the studies on finite-time synchronization of dynamical network have been mainly focused on static networks.

In addition, as bounded control of complex system is relevant in practical applications, for example, power networks cannot achieve complete synchronization, so it is desirable to obtain conditions within given bound such that the rotor phase differences between the generators remain [16]. In recent years, bounded control of complex dynamical network concerns the existence of bounded synchronization region globally stabilizing the complex system, which has grown very quickly, and it is one of the important issues in control theory and applications [17-21].

In [1-17], the authors studied the numbers of the nodes in the networks invariant about synchronization of complex dynamical networks. As the structures of many complex systems are typically dynamic, some new nodes can enter the network as time goes on [22]. Therefore, how to achieve synchronization of a growing dynamical network with bounded error is a very interesting and indeed important subject for research. Motivated by the existing works, the aim of this paper is to discuss finite-time synchronization of the 
growing complex dynamical network with nondelayed and delayed coupling. A new finite-time stabilization theory is proposed to investigate finite-time bounded synchronization of complex dynamical networks. Finite-time stabilization theory presented in this paper extends the conclusions of literatures [11-15] using finite-time stabilization theory.

The rest of this paper is organized as follows: Section 2 presents model and preliminaries. Section 3 gives the sufficient conditions of finite-time bounded synchronization. Section 4 presents an example and relates simulation results. Section 5 gives the conclusions of this paper.

\section{Model and Preliminaries}

Firstly, we introduce the network model and give some useful mathematical preliminaries.

Considering the growing complex dynamical network consisting of $N_{t}$ identical coupled nodes at time $t$, with each node being an $n$-dimensional dynamical system. The state equations of complex dynamical network can be described by

$$
\begin{aligned}
\frac{d x_{i}(t)}{d t}= & f\left(x_{i}(t), t\right)+c_{1} \sum_{j=1}^{N_{t}} a_{i j}(t) x_{j}(t) \\
& +c_{2} \sum_{j=1}^{N_{t}} b_{i j}(t) x_{j}(t-\tau), \quad i=1,2, \ldots, N_{t},
\end{aligned}
$$

where $x_{i}(t)=\left(x_{i 1}(t), x_{i 2}(t), \ldots, x_{i n}(t)\right)^{T}, f\left(x_{i}(t), t\right)$ standing for the activity of an individual subsystem is a vector value function, and $c_{1}$ and $c_{2}$ are two coupling strengths of the network. If there exists a link from node $i$ to $j(i \neq j)$ at time $t$, and $a_{i i}(t)=-\sum_{j=1, j \neq i}^{N_{t}} a_{i j}(t), b_{i i}(t)=-\sum_{j=1, j \neq i}^{N_{t}} b_{i j}(t)$.

Then the controlled dynamical network can be

$$
\begin{aligned}
\frac{d y_{i}(t)}{d t}= & f\left(y_{i}(t), t\right)+c_{1} \sum_{j=1}^{N_{t}} a_{i j}(t) y_{j}(t) \\
& +c_{2} \sum_{j=1}^{N_{t}} b_{i j}(t) y_{j}(t-\tau)+u_{i}(t) .
\end{aligned}
$$

So, the error equation is

$$
\begin{aligned}
\frac{d e_{i}(t)}{d t}= & f\left(y_{i}(t), t\right)-f\left(x_{i}(t), t\right)+c_{1} \sum_{j=1}^{N_{t}} a_{i j}(t) e_{j}(t) \\
& +c_{2} \sum_{j=1}^{N_{t}} b_{i j}(t) e_{j}(t-\tau)+u_{i},
\end{aligned}
$$

where $e_{i}(t)=\left(e_{i 1}(t), e_{i 2}(t), \ldots, e_{i n}(t)\right)^{T}, e_{i}(t)=y_{i}(t)-x_{i}(t)$.

We give the following preliminaries for obtaining the main result.

Assumption 1. We assume that $f$ is Lipschitz with respect to its argument; that is,

$$
\left\|f\left(y_{i}(t)\right)-f\left(x_{i}(t)\right)\right\| \leq \mu\left(y_{i}(t)-x_{i}(t)\right), \quad \mu \in R^{+} .
$$

Lemma 2 (see [23]). The following matrix inequality holds: $2 x^{T} y \leq x^{T} Q x+y^{T} Q^{-1} y$ for any vectors $x, y \in R^{m}$ and positive-definite matrix $Q \in R^{m \times m}$. If not specified otherwise, inequality $Q>0(Q<0, Q \geq 0, Q \leq 0)$ means $Q$ is a positive (or negative, or semipositive, or seminegative) definite matrix.

Lemma 3 (see [24]). Let $a_{1}, a_{2}, \ldots, a_{n}>0$ and $0<r<p$. Then $\left(\sum_{i=1}^{n} a_{i}^{p}\right)^{1 / p} \leq\left(\sum_{i=1}^{n} a_{i}^{r}\right)^{1 / r}$.

Lemma 4 (see [25]). Given the following dynamical system

$$
\dot{x}=f(x(t)), \quad f(0)=0, x \in R^{n}, x(0)=x_{0} .
$$

Assume that a continuous and positive-definite $V(x)$ satisfies the following differential inequality:

$$
\dot{V}(x) \leq-l V(x)-k V^{\eta}(x), \quad l>0, k>0,0<\eta<1 .
$$

Then, the origin of the dynamical system (5) is finite-time stable. The settling time $T_{x}\left(x_{0}\right)$ satisfies

$$
T_{x}\left(x_{0}\right) \leq \frac{1}{l(1-\eta)} \ln \left(1+\frac{l}{k} V^{1-\eta}\left(x_{0}\right)\right) .
$$

In this paper, to illustrate finite-time bounded stability further, we present the following theorem.

Theorem 5. Suppose that there exist continuous and positivedefinite $V(x), l>0,0<\eta<1, k>0$, and $0<\beta<\infty$ such that

$$
\dot{V}(x) \leq-l V(x)-k V^{\eta}(x)+\beta .
$$

Then, the trajectory of system (5) is finite-time stable, and

(i) if $V^{\eta}(x)>\beta /(1-\theta) k$, then $T \leq(1 / l(1-\eta)) \ln (1+$ $\left.\left(l / \theta_{0} k\right) V^{1-\eta}\left(x_{0}\right)\right), 0<\theta \leq 1,0<\theta_{0}<1 ;$

(ii) if $V(x)>\beta /(1-\theta) l$, then $T \leq\left(1 / l \theta_{0}(1-\eta)\right) \ln (1+$ $\left.\left(l \theta_{0} / k\right) V^{1-\eta}\left(x_{0}\right)\right), 0<\theta \leq 1,0<\theta_{0}<1$.

Proof. (i) Obviously, there are $0<\theta \leq 1$ such that inequality (8) can be

$$
\dot{V}(x) \leq-l V(x)-\theta k V^{\eta}(x)-(1-\theta) k V^{\eta}(x)+\beta .
$$

When $V^{\eta}(x)>\beta /(1-\theta) k, \dot{V}(x) \leq-l V(x)-\theta k V^{\eta}(x)$. According to Lemma 4, the decrease of $V(x)$ in finite time drives the trajectories of the dynamical system into $V^{\eta}(x) \leq$ $\beta /(1-\theta) k$. So, the trajectories of the dynamical system are bounded in finite time as

$$
\lim _{\theta \rightarrow \theta_{0}} x \in\left(V^{\eta}(x) \leq \frac{\beta}{(1-\theta) k}\right), \quad 0<\theta_{0}<1 .
$$

And the time needed to reach (10) is bounded as

$$
T \leq \frac{1}{l(1-\eta)} \ln \left(1+\frac{l}{\theta_{0} k} V^{1-\eta}\left(x_{0}\right)\right) .
$$

(ii) Similarly to (i), there are $0<\theta \leq 1$ such that inequality (8) can be

$$
\dot{V}(x) \leq-\theta l V(x)-k V^{\eta}(x)-(1-\theta) l V(x)+\beta .
$$


When $V(x)>\beta /(1-\theta) l, \dot{V}(x) \leq-\theta l V(x)-k V^{\eta}(x)$. Therefore, the decrease of $V(x)$ in finite time drives the trajectories of the dynamical system into $V(x) \leq \beta /(1-\theta) l$, and the trajectories of the dynamical system are bounded in finite time as

$$
\lim _{\theta \rightarrow \theta_{0}} x \in\left(V(x) \leq \frac{\beta}{(1-\theta) l}\right), \quad 0<\theta_{0}<1 .
$$

So, the time needed to reach (13) is bounded as

$$
T \leq \frac{1}{l \theta_{0}(1-\eta)} \ln \left(1+\frac{l \theta_{0}}{k} V^{1-\eta}\left(x_{0}\right)\right) .
$$

Remark 6. Let $V(x(t))=(1 / 2) e^{T}(x(t)) e(x(t))$, based on Theorem 5, we have the following conclusion for the settling time $T_{x}\left(x_{0}\right)$ : (i) $V^{\eta}(x(t))=\left((1 / 2)\|e(x(t))\|^{2}\right)^{\eta} \leq \beta /\left(1-\theta_{0}\right) k$; that is, $\|e(x(t))\| \leq \sqrt{2}\left[\beta /\left(1-\theta_{0}\right) k\right]^{1 / 2 \eta}$, so the error $e(x(t))$ is bounded; (ii) $V(x(t))=(1 / 2)\|e(x(t))\|^{2} \leq \beta /\left(1-\theta_{0}\right) l$, that is, $\|e(x(t))\| \leq \sqrt{2 \beta /\left(1-\theta_{0}\right) l}$, so the error $e(x(t))$ is also bounded.

Remark 7. When $\beta=0$, Theorem 5 is reduced to Lemma 4 .

Remark 8. In [26], the authors present new finite-time stability by turning $\dot{V}(t) \leq l V(t)-k V^{\eta}(t)$ into $\dot{V}(t) \leq l(t) V(t)-$ $k(t) V^{\eta}(t)$; the obtained results have less conservatism than the existing one. In this paper, as $\ln \left(1+\left(l / \theta_{0} k\right) V^{1-\eta}\left(x_{0}\right)\right) \neq$ $\ln \left(1+(l / k) V^{1-\eta}\left(x_{0}\right)\right)$, so $T \neq T_{x}$, that is, the convergence time of Theorem 5 can be different from Lemma 4 by turning $\dot{V}(x) \leq-l V(x)-k V^{\eta}(x)$ into $\dot{V}(x) \leq-l V(x)-k V^{\eta}(x)+\beta$, and $\beta$ is adjustable parameter.

Remark 9. In [27], the authors present new inequality theorems by turning $\dot{V}(t) \leq-l(t) V(t)+f(t)$ into $\lim _{t \rightarrow \infty} V(t) \leq$ $b / a_{2}$, and $V(t)$ is bounded for $t \rightarrow \infty$. In this paper, $V(t)$ is bounded for $t \rightarrow T$ by using $\dot{V}(x) \leq-l V(x)-k V^{\eta}(x)+\beta$; T is finite-time.

\section{Finite-Time Bounded Synchronization of Complex Networks}

In this section, we study finite-time bounded synchronization between two networks. We can give the following main result.

Theorem 10. If Assumption 1 holds, and there exists

$$
\begin{aligned}
\gamma> & \mu+\frac{l}{2}+\frac{c_{1} N_{t}\left(\mu_{1}^{2} \lambda_{\max }\left\{Q_{1}\right\}+\lambda_{\max }\left\{Q_{1}^{-1}\right\}\right)}{2} \\
+ & \frac{c_{2} N_{t} \mu_{2}^{2} \lambda_{\max }\left\{Q_{2}\right\}}{2}, \quad c_{2}=\frac{1}{\phi\left(\|e(t-\tau)\|^{2}+\alpha\right)},
\end{aligned}
$$

where $\alpha>0, c_{1}>0, \phi>0, e(t-\tau)=\left(\left(e_{1}(t-\tau)\right)^{T}, \ldots,\left(e_{N_{t}}(t-\right.\right.$ $\left.\tau))^{T}\right)^{T},\left|a_{i j}(t)\right| \leq \mu_{1} \in R$, and $\left|b_{i j}(t)\right| \leq \mu_{2} \in R$.
Then complex dynamical networks (1)-(2) can realize finite-time bounded synchronization based on the following controller:

$$
u_{i}=-\gamma e_{i}(t)-\rho \operatorname{sign}\left(e_{i}(t)\right)\left|e_{i}(t)\right|^{\xi},
$$

where $\rho=k / 2^{\eta}, \eta=(\xi+1) / 2$.

Proof. According to Theorem 5, we only need to prove $\dot{V}(x)+$ $l V(x)+k V^{\eta}(x) \leq \beta$.

Considering the following nonnegative function:

$$
V(t)=\frac{1}{2} \sum_{i=1}^{N_{t}}\left(e_{i}(t)\right)^{T} e_{i}(t)
$$

we have

$$
\begin{aligned}
& \dot{V}(x)+l V(x)+k V^{\eta}(x)=\sum_{i=1}^{N_{t}}\left(e_{i}(t)\right)^{T}\left[f\left(y_{i}(t), t\right)\right. \\
& -f\left(x_{i}(t), t\right)+c_{1} \sum_{j=1}^{N_{t}} a_{i j}(t) e_{j}(t) \\
& +c_{2} \sum_{j=1}^{N_{t}} b_{i j}(t) e_{j}(t-\tau)-\gamma e_{i}(t) \\
& \left.-\rho \operatorname{sign}\left(e_{i}(t)\right)\left|e_{i}(t)\right|^{\xi}\right]+\frac{l}{2} \sum_{i=1}^{N_{t}}\left(e_{i}(t)\right)^{T} e_{i}(t) \\
& +k\left(\frac{1}{2} \sum_{i=1}^{N_{t}}\left(e_{i}(t)\right)^{T} e_{i}(t)\right)^{\eta}=\sum_{i=1}^{N_{t}}\left(e_{i}(t)\right)^{T} \\
& \cdot\left[f\left(y_{i}(t), t\right)-f\left(x_{i}(t), t\right)\right] \\
& +c_{1} \sum_{i=1}^{N_{t}} \sum_{j=1}^{N_{t}} a_{i j}(t)\left(e_{i}(t)\right)^{T} e_{j}(t) \\
& +c_{2} \sum_{i=1}^{N_{t}} \sum_{j=1}^{N_{t}} b_{i j}(t)\left(e_{i}(t)\right)^{T} e_{j}(t-\tau)-\rho \sum_{i=1}^{N_{t}}\left(e_{i}(t)\right)^{T} \\
& \cdot \operatorname{sign}\left(e_{i}(t)\right)\left|e_{i}(t)\right|^{\xi}-\gamma \sum_{i=1}^{N_{t}}\left(e_{i}(t)\right)^{T} e_{i}(t)+\frac{l}{2} \\
& \cdot \sum_{i=1}^{N_{t}}\left(e_{i}(t)\right)^{T} e_{i}(t)+k\left(\frac{1}{2} \sum_{i=1}^{N_{t}}\left(e_{i}(t)\right)^{T} e_{i}(t)\right)^{\eta} .
\end{aligned}
$$

In (18), by using Lemma 2, obviously,

$$
\begin{aligned}
\sum_{i=1}^{N_{t}} \sum_{j=1}^{N_{t}} a_{i j}(t)\left[e_{i}(t)\right]^{T} e_{j}(t) \\
\leq \frac{1}{2} \sum_{i=1}^{N_{t}} \sum_{j=1}^{N_{t}} a_{i j}^{2}(t)\left[e_{i}(t)\right]^{T} Q_{1} e_{i}(t) \\
\quad+\frac{1}{2} \sum_{i=1}^{N_{t}} \sum_{j=1}^{N_{t}}\left(e_{j}(t)\right)^{T} Q_{1}^{-1} e_{j}(t)
\end{aligned}
$$




$$
\begin{aligned}
\leq & \frac{N_{t} \mu_{1}^{2}}{2} \sum_{i=1}^{N_{t}}\left[e_{i}(t)\right]^{T} Q_{1} e_{i}(t) \\
& +\frac{N_{t}}{2} \sum_{j=1}^{N_{t}}\left(e_{j}(t)\right)^{T} Q_{1}^{-1} e_{j}(t),
\end{aligned}
$$

$\sum_{i=1}^{N_{t}} \sum_{j=1}^{N_{t}} b_{i j}(t)\left[e_{i}(t)\right]^{T} e_{j}(t-\tau)$

$\leq \frac{1}{2} \sum_{i=1}^{N_{t}} \sum_{j=1}^{N_{t}} b_{i j}^{2}(t)\left[e_{i}(t)\right]^{T} Q_{2} e_{i}(t)$

$+\frac{1}{2} \sum_{i=1}^{N_{t}} \sum_{j=1}^{N_{t}}\left(e_{j}(t-\tau)\right)^{T} Q_{2}^{-1} e_{j}(t-\tau)$

$\leq \frac{N_{t} \mu_{2}^{2}}{2} \sum_{i=1}^{N_{t}}\left[e_{i}(t)\right]^{T} Q_{2} e_{i}(t)$

$+\frac{N_{t}}{2} \sum_{j=1}^{N_{t}}\left(e_{j}(t-\tau)\right)^{T} Q_{2}^{-1} e_{j}(t-\tau)$,

(19)

where $\left|a_{i j}(t)\right| \leq \mu_{1} \in R,\left|b_{i j}(t)\right| \leq \mu_{2} \in R$.

So,

$$
\begin{aligned}
& \dot{V}(x)+l V(x)+k V^{\eta}(x) \\
& \leq \sum_{i=1}^{N_{t}}\left(e_{i}(t)\right)^{T}\left[f\left(y_{i}(t), t\right)-f\left(x_{i}(t), t\right)\right] \\
& +\frac{c_{1} N_{t} \mu_{1}^{2}}{2} \sum_{j=1}^{N_{t}}\left[e_{i}(t)\right]^{T} Q_{1} e_{i}(t)+\frac{c_{1} N_{t}}{2} \\
& \cdot \sum_{j=1}^{N_{t}}\left(e_{j}(t)\right)^{T} Q_{1}^{-1} e_{j}(t)+\frac{c_{2} N_{t} \mu_{2}^{2}}{2} \\
& \cdot \sum_{i=1}^{N_{t}}\left[e_{i}(t)\right]^{T} Q_{2} e_{i}(t)+\frac{c_{2} N_{t}}{2} \sum_{j=1}^{N_{t}}\left(e_{j}(t-\tau)\right)^{T} Q_{2}^{-1} \\
& \cdot e_{j}(t-\tau)-\rho \sum_{i=1}^{N_{t}}\left(e_{i}(t)\right)^{T} \operatorname{sign}\left(e_{i}(t)\right)\left|e_{i}(t)\right|^{\xi} \\
& -\gamma \sum_{i=1}^{N_{t}}\left(e_{i}(t)\right)^{T} e_{i}(t)+\frac{l}{2} \sum_{i=1}^{N_{t}}\left(e_{i}(t)\right)^{T} e_{i}(t) \\
& +k\left(\frac{1}{2} \sum_{i=1}^{N_{t}}\left(e_{i}(t)\right)^{T} e_{i}(t)\right)^{\eta} \leq \mu \sum_{i=1}^{N_{t}}\left(e_{i}(t)\right)^{T} e_{i}(t) \\
& +\frac{c_{1} N_{t} \mu_{1}^{2} \sum_{i=1}^{N_{t}}\left[e_{i}(t)\right]^{T} Q_{1} e_{i}(t)+\frac{c_{1} N_{t}}{2}}{2}
\end{aligned}
$$

$$
\begin{aligned}
& \cdot \sum_{j=1}^{N_{t}}\left(e_{j}(t)\right)^{T} Q_{1}^{-1} e_{j}(t)+\frac{c_{2} N_{t} \mu_{2}^{2}}{2} \\
& \cdot \sum_{i=1}^{N_{t}}\left[e_{i}(t)\right]^{T} Q_{2} e_{i}(t)+\frac{c_{2} N_{t}}{2} \sum_{j=1}^{N_{t}}\left(e_{j}(t-\tau)\right)^{T} Q_{2}^{-1}
\end{aligned}
$$$$
\cdot e_{j}(t-\tau)-\rho \sum_{i=1}^{N_{t}}\left(e_{i}(t)\right)^{T} \operatorname{sign}\left(e_{i}(t)\right)\left|e_{i}(t)\right|^{\xi}
$$$$
-\gamma \sum_{i=1}^{N_{t}}\left(e_{i}(t)\right)^{T} e_{i}(t)+\frac{l}{2} \sum_{i=1}^{N_{t}}\left(e_{i}(t)\right)^{T} e_{i}(t)
$$$$
+k\left(\frac{1}{2} \sum_{i=1}^{N_{t}}\left(e_{i}(t)\right)^{T} e_{i}(t)\right)^{\eta} \leq \mu \sum_{i=1}^{N_{t}}\left(e_{i}(t)\right)^{T} e_{i}(t)
$$$$
+\frac{c_{1} N_{t} \mu_{1}^{2} \lambda_{\max }\left\{Q_{1}\right\}}{2} \sum_{i=1}^{N_{t}}\left[e_{i}(t)\right]^{T} e_{i}(t)
$$$$
+\frac{c_{1} N_{t} \lambda_{\max }\left\{Q_{1}^{-1}\right\}}{2} \sum_{j=1}^{N_{t}}\left(e_{j}(t)\right)^{T} e_{j}(t)
$$$$
+\frac{c_{2} N_{t} \mu_{2}^{2} \lambda_{\max }\left\{Q_{2}\right\}}{2} \sum_{i=1}^{N_{t}}\left[e_{i}(t)\right]^{T} e_{i}(t)
$$

$$
+\frac{c_{2} N_{t} \lambda_{\max }\left\{Q_{2}^{-1}\right\}}{2} \sum_{j=1}^{N_{t}}\left(e_{j}(t-\tau)\right)^{T} e_{j}(t-\tau)
$$$$
-\rho \sum_{i=1}^{N_{t}}\left(e_{i}(t)\right)^{T} \operatorname{sign}\left(e_{i}(t)\right)\left|e_{i}(t)\right|^{\xi}
$$$$
-\gamma \sum_{i=1}^{N_{t}}\left(e_{i}(t)\right)^{T} e_{i}(t)+\frac{l}{2} \sum_{i=1}^{N_{t}}\left(e_{i}(t)\right)^{T} e_{i}(t)
$$$$
+k\left(\frac{1}{2} \sum_{i=1}^{N_{t}}\left(e_{i}(t)\right)^{T} e_{i}(t)\right)^{\eta} \text {. }
$$

In (20), obviously,

$$
\begin{aligned}
& \sum_{i=1}^{N_{t}}\left(e_{i}(t)\right)^{T} \operatorname{sign}\left(e_{i}(t)\right)\left|e_{i}(t)\right|^{\xi} \\
& =\sum_{i=1}^{N_{t}}\left(\left|e_{i}(t)\right|^{\xi}\right)^{T} \operatorname{sign}\left(e_{i}(t)\right) e_{i}(t) \\
& =\sum_{i=1}^{N_{t}}\left(\left|e_{i}(t)\right|^{\xi}\right)^{T}\left|e_{i}(t)\right|=\sum_{i=1}^{N_{t}} \sum_{j=1}^{N_{t}}\left|e_{i j}(t)\right|^{\xi} .
\end{aligned}
$$

By Lemma 3, we have

$$
\left(\sum_{i=1}^{N_{t}} \sum_{j=1}^{N_{t}}\left|e_{i j}\right|^{\xi+1}\right)^{1 /(\xi+1)} \geq\left(\sum_{i=1}^{N_{t}} \sum_{j=1}^{N_{t}}\left|e_{i j}\right|^{2}\right)^{1 / 2} .
$$


Hence,

$$
\begin{aligned}
\sum_{i=1}^{N_{t}} \sum_{j=1}^{N_{t}}\left|e_{i j}(t)\right|^{\xi+1} & \geq\left(\sum_{i=1}^{N_{t}} \sum_{j=1}^{N_{t}}\left|e_{i j}\right|^{2}\right)^{(\xi+1) / 2} \\
& =\left(\sum_{i=1}^{N_{t}}\left(e_{i}(t)\right)^{T} e_{i}(t)\right)^{(\xi+1) / 2} .
\end{aligned}
$$

So

$$
\begin{aligned}
& \dot{V}(x)+l V(x)+k V^{\eta}(x) \leq \mu \sum_{i=1}^{N_{t}}\left(e_{i}(t)\right)^{T} e_{i}(t) \\
& +\frac{c_{1} N_{t} \mu_{1}^{2} \lambda_{\max }\left\{Q_{1}\right\}}{2} \sum_{i=1}^{N_{t}}\left[e_{i}(t)\right]^{T} e_{i}(t) \\
& +\frac{c_{1} N_{t} \lambda_{\max }\left\{Q_{1}^{-1}\right\}}{2} \sum_{j=1}^{N_{t}}\left(e_{j}(t)\right)^{T} e_{j}(t) \\
& +\frac{c_{2} N_{t} \mu_{2}^{2} \lambda_{\max }\left\{Q_{2}\right\}}{2} \sum_{i=1}^{N_{t}}\left[e_{i}(t)\right]^{T} e_{i}(t) \\
& +\frac{c_{2} N_{t} \lambda_{\max }\left\{Q_{2}^{-1}\right\}}{2} \sum_{j=1}^{N_{t}}\left(e_{j}(t-\tau)\right)^{T} e_{j}(t-\tau) \\
& -\rho\left(\sum_{i=1}^{N_{t}}\left(e_{i}(t)\right)^{T} e_{i}(t)\right)^{(\xi+1) / 2}-\gamma \sum_{i=1}^{N_{t}}\left(e_{i}(t)\right)^{T} e_{i}(t) \\
& +\frac{l}{2} \sum_{i=1}^{N_{t}}\left(e_{i}(t)\right)^{T} e_{i}(t)+k\left(\frac{1}{2} \sum_{i=1}^{N_{t}}\left(e_{i}(t)\right)^{T} e_{i}(t)\right)^{\eta} \\
& \leq \sum_{i=1}^{N_{t}}\left[\mu+\frac{l}{2}+\frac{c_{1} N_{t}\left(\mu_{1}^{2} \lambda_{\max }\left\{Q_{1}\right\}+\lambda_{\max }\left\{Q_{1}^{-1}\right\}\right)}{2}\right. \\
& \left.+\frac{c_{2} N_{t} \mu_{2}^{2} \lambda_{\max }\left\{Q_{2}\right\}}{2}-\gamma\right]\left(e_{i}(t)\right)^{T} e_{i}(t) \\
& -\rho\left(\sum_{i=1}^{N_{t}}\left(e_{i}(t)\right)^{T} e_{i}(t)\right)^{(\xi+1) / 2}+k\left(\frac{1}{2}\right. \\
& \left.\cdot \sum_{i=1}^{N_{t}}\left(e_{i}(t)\right)^{T} e_{i}(t)\right)^{\eta}+\frac{c_{2} N_{t} \lambda_{\max }\left\{Q_{2}^{-1}\right\}}{2} \\
& \cdot \sum_{j=1}^{N_{t}}\left(e_{j}(t-\tau)\right)^{T} e_{j}(t-\tau) .
\end{aligned}
$$

By choosing appropriate $\gamma>\mu+l / 2+c_{1} N_{t}\left(\mu_{1}^{2} \lambda_{\max }\left\{Q_{1}\right\}+\right.$ $\left.\lambda_{\text {max }}\left\{Q_{1}^{-1}\right\}\right) / 2+c_{2} N_{t} \mu_{2}^{2} \lambda_{\text {max }}\left\{Q_{2}\right\} / 2, c_{2}=1 / \phi\left(\|e(t-\tau)\|^{2}+\alpha\right)$, $\rho=k / 2^{\eta}, \eta=(\xi+1) / 2$, then, $\dot{V}(x)+l V(x)+k V^{\eta}(x) \leq$ $N_{t} \lambda_{\max }\left\{Q_{2}^{-1}\right\} \sum_{j=1}^{N_{t}}\left(e_{j}(t-\tau)\right)^{T} e_{j}(t-\tau) / 2 \phi\left(\|e(t-\tau)\|^{2}+\alpha\right) \leq$ $N_{t} \lambda_{\max }\left\{Q_{2}^{-1}\right\} / 2 \phi=\beta$. Based on Theorem 5 and Remark 6, the error system is finite-time bounded stability. The proof is completed.
Remark 5. By Remark 6, if $V^{\eta}(x)>\beta /(1-\theta) k$, then $T \leq$ $(1 / l(1-\eta)) \ln \left(1+\left(l / \theta_{0} k\right) V^{1-\eta}\left(x_{0}\right)\right),\|e(x(T))\| \leq \sqrt{2}[\beta /(1-$ $\left.\left.\theta_{0}\right) k\right]^{1 / 2 \eta}$; if $V(x)>\beta /(1-\theta) l$, then $T \leq\left(1 / l \theta_{0}(1-\eta)\right) \ln (1+$ $\left.\left(l \theta_{0} / k\right) V^{1-\eta}\left(x_{0}\right)\right),\|e(x(T))\| \leq \sqrt{2 \beta /\left(1-\theta_{0}\right) l}$.

Remark 6. For the finite time $t_{0}$, we can find the appropriate value $\beta$ by adjusting $\phi$ value. (i) If $\phi \geq$ (1$\theta) k N_{t} \lambda_{\max }\left\{Q_{2}^{-1}\right\} / 2 V^{\eta}\left(t_{0}\right)$, then $\beta=N_{t} \lambda_{\max }\left\{Q_{2}^{-1}\right\} / 2 \phi$ and $V^{\eta}\left(t_{0}\right)>\beta /(1-\theta) k$. (ii) If $\phi \geq(1-\theta) l N_{t} \lambda_{\max }\left\{Q_{2}^{-1}\right\} / 2 V\left(t_{0}\right)$, then $\beta=N_{t} \lambda_{\text {max }}\left\{Q_{2}^{-1}\right\} / 2 \phi$ and $V\left(t_{0}\right)>\beta /(1-\theta) l$.

Remark 7. In the network, when the number of nodes $N_{t}$ increases, the value of $\gamma$ will also increase, and the suitable $\gamma$ value can always be found.

\section{Illustrative Example}

In this section, we present an example to illustrate the usefulness of Theorem 10 in this paper.

Consider the following Lü system [28, 29]:

$$
\begin{aligned}
\dot{x}^{i} & =\left(\begin{array}{ccc}
-36 & 36 & 0 \\
0 & 20 & 0 \\
0 & 0 & -3
\end{array}\right)\left(\begin{array}{c}
x_{1}^{i} \\
x_{2}^{i} \\
x_{3}^{i}
\end{array}\right)+\left(\begin{array}{c}
0 \\
-x_{1}^{i} x_{3}^{i} \\
x_{1}^{i} x_{2}^{i}
\end{array}\right) \\
& =G x^{i}+h\left(x^{i}\right) .
\end{aligned}
$$

Obviously, $\|G\|_{2} \approx 52.9843$, and

$$
\begin{aligned}
& \left\|h\left(y^{i}\right)-h\left(x^{i}\right)\right\|_{2} \\
& \quad \leq \sqrt{\left(-y_{3}^{i} e_{1}^{i}-x_{1}^{i} e_{3}^{i}\right)^{2}+\left(y_{2}^{i} e_{1}^{i}+x_{1}^{i} e_{2}^{i}\right)^{2}} \\
& \quad \leq \sqrt{2 M_{1}^{2}+M_{2}^{2}+M_{3}^{2}}\left\|e^{i}\right\|_{2} \approx 64.6142\left\|e^{i}\right\|_{2} .
\end{aligned}
$$

It is well known that Lü attractor is bounded. Here we suppose that all nodes are running in the given bounded region [30]. Our theoretical and numerical analyses show that there exist constants $M_{1}=25, M_{2}=30$, and $M_{3}=45$ satisfying $\left\|x_{j}^{i}\right\|,\left\|y_{j}^{i}\right\| \leq M_{j}, j=1,2,3$, so

$$
\left\|f\left(y^{i}\right)-f\left(x^{i}\right)\right\|_{2} \leq \mu\left\|e^{i}\right\|_{2} \approx 117.5985\left\|e^{i}\right\|_{2} .
$$

In numerical simulation, let the initial values of the state variable be rand $[0,3]$. When the time $0<t \leq 0.04$, the coupling network with 6 nodes is described by the coupling matrices $A, B$,

$A$

$$
=\left(\begin{array}{cccccc}
-4+\sin t & 1 & 1 & 1 & 1 & -\sin t \\
1 & -4 & 0 & 1 & 1 & 1 \\
1 & 0 & -3 & 1 & 1 & 0 \\
1 & 1 & 1 & -3 & 0 & 0 \\
1 & 1 & 1 & 0 & -3-\sin t & \sin t \\
-\sin t & 1 & 0 & 0 & \sin t & -1
\end{array}\right),
$$


$B$

$$
=\left(\begin{array}{cccccc}
-3-\cos ^{2} t & 1 & 0 & 2 & 0 & \cos ^{2} t \\
1 & -3 & 0 & 1 & 0 & 1 \\
0 & 0 & -3 & 1 & 1 & 1 \\
2 & 1 & 1 & -5 & 1 & 0 \\
0 & 0 & 1 & 1 & -\sin ^{2} t-2 & \sin ^{2} t \\
\cos ^{2} t & 1 & 1 & 0 & \sin ^{2} t & -3
\end{array}\right) .
$$

Suppose that there are new nodes entering the network at time $0.04<t \leq 0.08$, the coupling network with 7 nodes is described by the coupling matrices $A^{\prime}, B^{\prime}$,

$$
\begin{aligned}
& A^{\prime} \\
& =\left(\begin{array}{ccccccc}
\cos t-1 & -\cos t & 0 & 2 & 0 & -1 & 0 \\
-\cos t & \cos t & 4 & 0 & 0 & -2 & -2 \\
0 & 4 & 1 & -2 & -3 & 0 & 0 \\
2 & 0 & -2 & -4 & 3 & 0 & 1 \\
0 & 0 & -3 & 3 & -2 & 2 & 0 \\
-1 & -2 & 0 & 0 & 2 & 1+\sin t & -\sin t \\
0 & -2 & 0 & 1 & 0 & -\sin t & 1+\sin t
\end{array}\right), \\
& B^{\prime}=\left(\begin{array}{ccccccc}
-\sin t & \sin t & -2 & 0 & 2 & 0 & 0 \\
\sin t & -1 & 0 & 0 & 0 & 0 & 1-\sin t \\
-2 & 0 & 1 & 2 & -1 & 0 & 0 \\
0 & 0 & 2 & -4 & 0 & 2 & 0 \\
2 & 0 & -1 & 0 & 0 & 0 & -1 \\
0 & 0 & 0 & 2 & 0 & -3 & 1 \\
0 & 1-\sin t & 0 & 0 & -1 & 1 & -1+\sin t
\end{array}\right)
\end{aligned}
$$

Let $k=l=1, \alpha=0.1, c_{1}=1, \tau=0.05, Q_{1}=Q_{2}=I, \eta=0.75$, $\xi=0.5, c_{2}=1 / 100\left(\|e(t-\tau)\|^{2}+0.1\right), \gamma_{1}>196.0985+108 c_{2}$ (network with 6 nodes), $\gamma_{2}>177.5985+63 c_{2}$ (network with 7 nodes); let $\gamma=300+108 c_{2}$. Let $E_{j}(t)=$ $\sqrt{\sum_{i=1}^{N_{t}}\left\|y_{i j}(t)-x_{i j}(t)\right\|^{2} / N_{t}}(j=1,2,3)$, obviously, two networks achieve synchronization when $E_{j}(t)$ no longer increases. Figure 1 shows the variance of the synchronization errors. Figure 1 shows $E_{j}(t) \rightarrow 0$. Numerical simulation illustrates the effectiveness of Theorem 10.

\section{Conclusion}

In this paper, finite-time synchronization for a class of the growing complex dynamical network with nondelayed and delayed coupling was investigated. A new finite-time bounded stable theory is proposed; synchronization criterion was derived to ensure the realization of finite-time bounded synchronization. Finally, numerical simulation was given to verify the effectiveness of the proposed schemes.
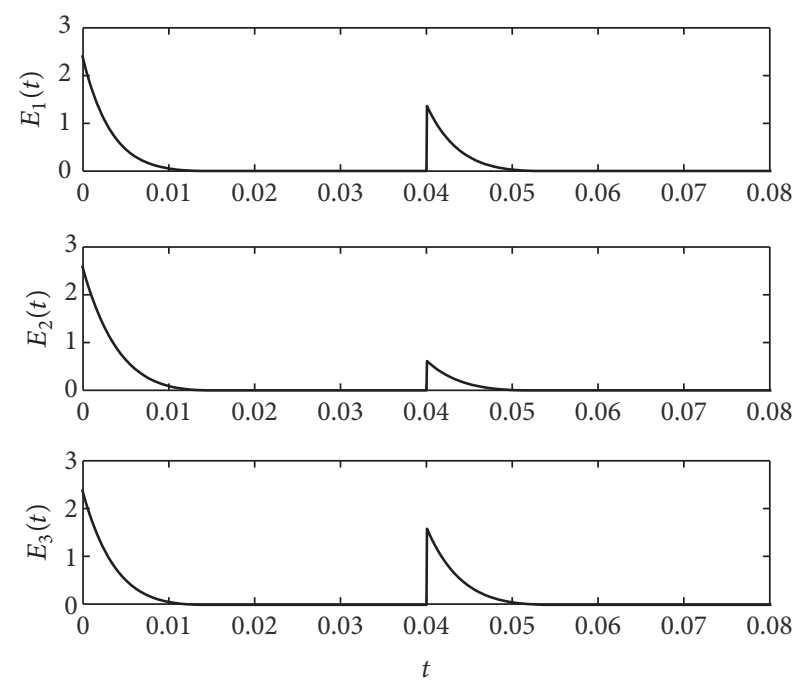

FIGURE 1: Finite-time bounded synchronization errors.

\section{Competing Interests}

The authors declare that they have no competing interests.

\section{Acknowledgments}

This work was supported by the National Natural Science Foundation of China (61673221, 61673257, and 11501367), the Youth Fund Project of the Humanities and Social Science Research for the Ministry of Education of China (14YJCZH173), Top-notch Academic Programs Project of Jiangsu Higher Education Institutions (Jiangsu Province Office, no. [2015]1, PPZY2015B104), the Major Natural Science Research Projects of Jiangsu Higher Education Institutions (12KJA630001), the Key Laboratory of Financial Engineering of Jiangsu Province (NSK2015-16), the Science and Technology Research Key Program for the Education Department of Hubei Province of China (D20156001), and Applied Economics Advantage Subject Construction Project of Jiangsu Higher Education Institutions (Jiangsu Province Office, no. [2014]37).

\section{References}

[1] J. Lü and G. Chen, "A time-varying complex dynamical network model and its controlled synchronization criteria," IEEE Transactions on Automatic Control, vol. 50, no. 6, pp. 841-846, 2005.

[2] J. Lu, J. Kurths, J. Cao, N. Mahdavi, and C. Huang, "Synchronization control for nonlinear stochastic dynamical networks: pinning impulsive strategy," IEEE Transactions on Neural Networks and Learning Systems, vol. 23, no. 2, pp. 285-292, 2012.

[3] J. Lu, J. Zhong, Y. Tang, T. Huang, J. Cao, and J. Kurths, "Synchronization in output-coupled temporal Boolean networks," Scientific Reports, vol. 4, article 6292, 2014.

[4] R. Lu, W. Yu, J. Lu, and A. Xue, "Synchronization on complex networks of networks," IEEE Transactions on Neural Networks and Learning Systems, vol. 25, no. 11, pp. 2110-2118, 2014.

[5] Y. Tang and W. K. Wong, "Distributed synchronization of coupled neural networks via randomly occurring control," IEEE 
Transactions on Neural Networks and Learning Systems, vol. 24, no. 3, pp. 435-447, 2013.

[6] Y. Tang, Z. Wang, H. Gao, S. Swift, and J. Kurths, "A constrained evolutionary computation method for detecting controlling regions of cortical networks," IEEE/ACM Transactions on Computational Biology and Bioinformatics, vol. 9, no. 6, pp. 15691581, 2012.

[7] Y. Tang, H. Gao, W. Zou, and J. Kurths, "Distributed synchronization in networks of agent systems with nonlinearities and random switchings," IEEE Transactions on Cybernetics, vol. 43, no. 1, pp. 358-370, 2013.

[8] J.-G. Dong and X. Xue, "Finite-time synchronization of Kuramoto-type oscillators," Nonlinear Analysis: Real World Applications, vol. 26, pp. 133-149, 2015.

[9] Y. Shen and X. Xia, "Semi-global finite-time observers for nonlinear systems," Automatica, vol. 44, no. 12, pp. 3152-3156, 2008.

[10] W. Cui, S. Sun, J.-a. Fang, Y. Xu, and L. Zhao, "Finite-time synchronization of Markovian jump complex networks with partially unknown transition rates," Journal of the Franklin Institute, vol. 351, no. 5, pp. 2543-2561, 2014.

[11] H. Shen, J. H. Park, and Z.-G. Wu, "Finite-time synchronization control for uncertain Markov jump neural networks with input constraints," Nonlinear Dynamics, vol. 77, no. 4, pp. 1709-1720, 2014.

[12] X. Zhang, G. Feng, and Y. Sun, "Finite-time stabilization by state feedback control for a class of time-varying nonlinear systems," Automatica, vol. 48, no. 3, pp. 499-504, 2012.

[13] X. Wang, J.-A. Fang, H. Mao, and A. Dai, "Finite-time global synchronization for a class of Markovian jump complex networks with partially unknown transition rates under feedback control," Nonlinear Dynamics, vol. 79, no. 1, pp. 47-61, 2015.

[14] X. Liu, X. Yu, and H. Xi, "Finite-time synchronization of neutral complex networks with Markovian switching based on pinning controller," Neurocomputing, vol. 153, pp. 148-158, 2015.

[15] T. Shi, "Finite-time control of linear systems under time-varying sampling," Neurocomputing, vol. 151, no. 3, pp. 1327-1331, 2015.

[16] D. Florian and B. Francesco, "Synchronization and transient stability in power networks and non-uniform kuramoto oscillators," in Proceedings of the American Control Conference, Baltimore, Md, USA, June 2010.

[17] S. Zhai and X.-S. Yang, "Bounded synchronisation of singularly perturbed complex network with an application to power systems," IET Control Theory \& Applications, vol. 8, no. 1, pp. 61-66, 2014.

[18] Y. Xu, J. Zhang, W. Zhou, and D. Tong, "Adaptive synchronization of complex dynamical networks with bounded delay feedback controller," Optik, vol. 131, pp. 467-474, 2017.

[19] C. Xie, Y. Xu, and D. Tong, "Chaos synchronization of financial chaotic system with external perturbation," Discrete Dynamics in Nature and Society, vol. 2015, Article ID 731376, 7 pages, 2015.

[20] Y. Xu, B. Li, W. Zhou, and J. Fang, "Mean square function synchronization of chaotic systems with stochastic effects," Nonlinear Dynamics, vol. 70, no. 1, pp. 289-294, 2012.

[21] K. Ding and X. Xu, "Chaotic synchronization of modified discrete-time Tinkerbell systems," Discrete Dynamics in Nature and Society, vol. 2016, Article ID 5218080, 7 pages, 2016.

[22] Z. Wu, X. Fu, and G. Chen, "Monitoring the topology of growing dynamical networks," International Journal of Modern Physics C, vol. 21, no. 8, pp. 1051-1063, 2010.
[23] Z. Wang, L. Huang, Y. Wang, and Y. Zuo, "Synchronization analysis of networks with both delayed and non-delayed couplings via adaptive pinning control method," Communications in Nonlinear Science and Numerical Simulation, vol. 15, no. 12, pp. 4202-4208, 2010.

[24] G. H. Hardy, J. E. Littlewood, and G. Polya, Inequalities, Cambridge University Press, Cambridge, UK, 1952.

[25] M. Jiang, S. Wang, J. Mei, and Y. Shen, "Finite-time synchronization control of a class of memristor-based recurrent neural networks," Neural Networks, vol. 63, pp. 133-140, 2015.

[26] Y. Xu, W. Zhou, J. Fang, C. Xie, and D. Tong, "Finite-time synchronization of the complex dynamical network with nonderivative and derivative coupling," Neurocomputing, vol. 173, pp. 1356-1361, 2016.

[27] Y. Xu, Y. Lu, W. Yan, W. Zhou, and J. Fang, "Bounded synchronization of the general complex dynamical network with delay feedback controller," Nonlinear Dynamics, vol. 84, no. 2, pp. 661667, 2016.

[28] J. Lü and G. Chen, "A new chaotic attractor coined," International Journal of Bifurcation and Chaos in Applied Sciences and Engineering, vol. 12, no. 3, pp. 659-661, 2002.

[29] W. Zhou, Y. Xu, H. Lu, and L. Pan, "On dynamics analysis of a new chaotic attractor," Physics Letters. A, vol. 372, no. 36, pp. 5773-5777, 2008.

[30] J. Zhou and J. Lu, “Topology identification of weighted complex dynamical networks," Physica A, vol. 386, no. 1, pp. 481-491, 2007. 


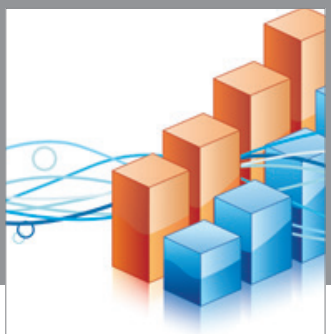

Advances in

Operations Research

vatem alat4

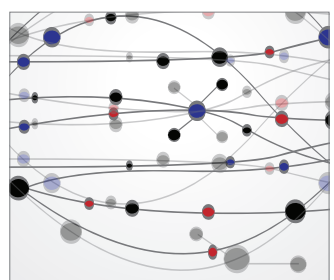

\section{The Scientific} World Journal
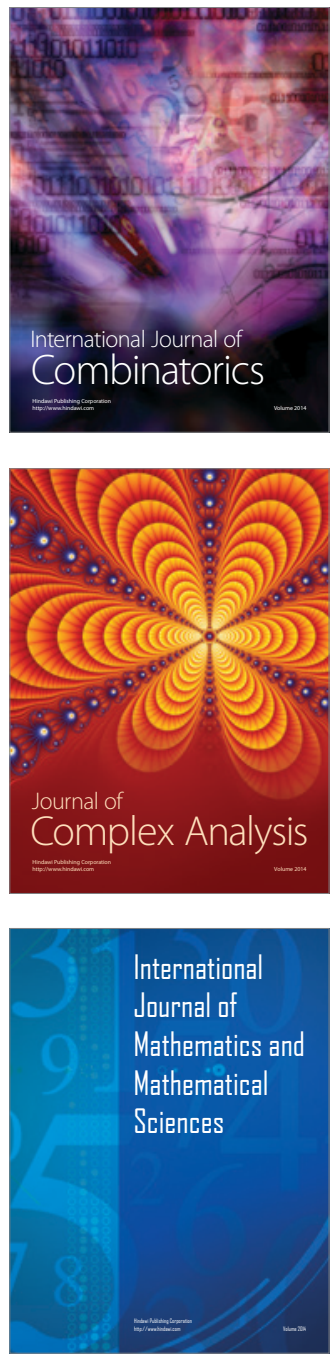
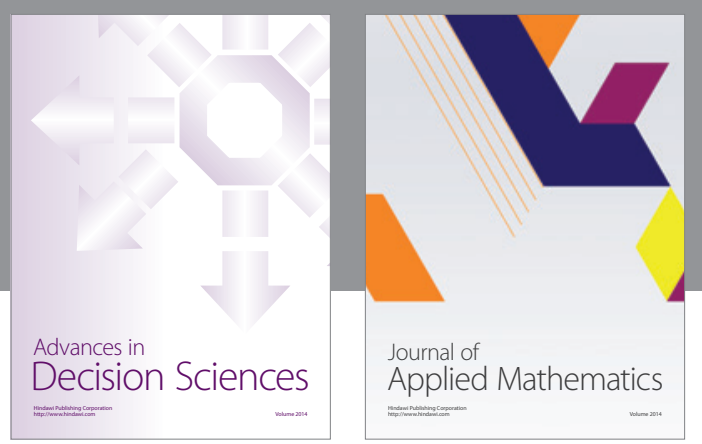

Algebra

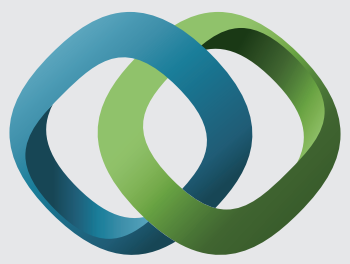

\section{Hindawi}

Submit your manuscripts at

https://www.hindawi.com
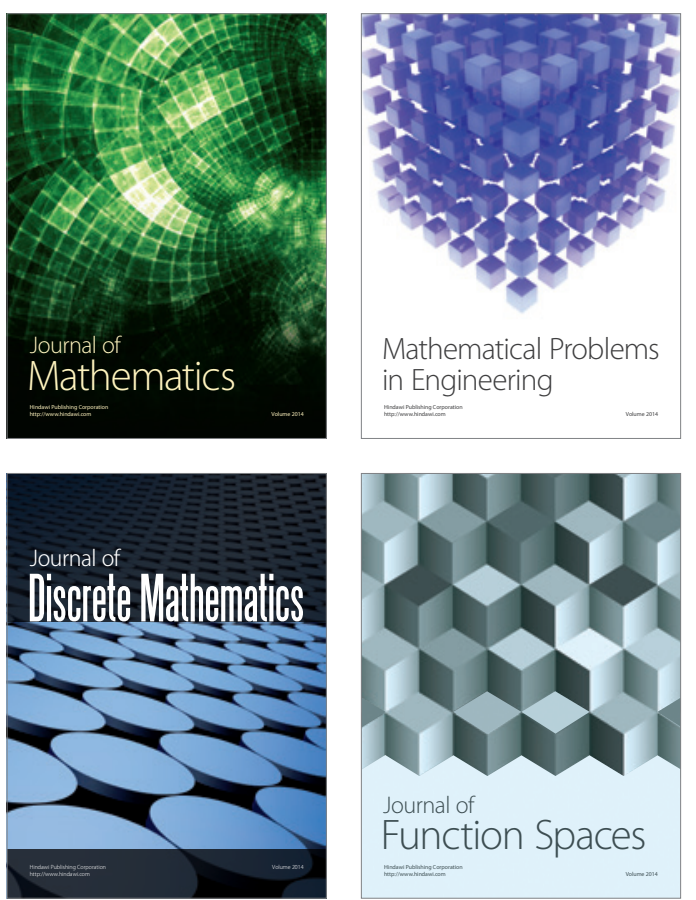

Mathematical Problems in Engineering
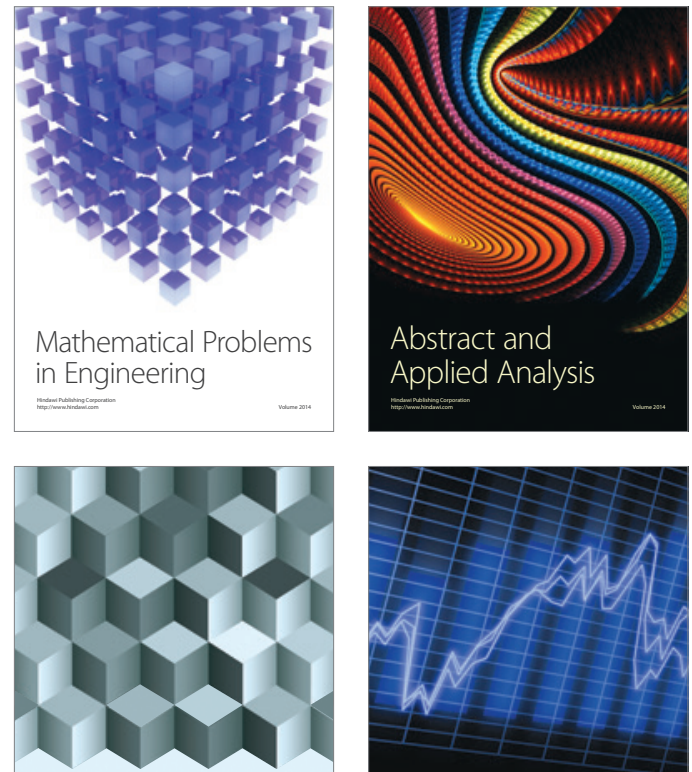

Journal of

Function Spaces

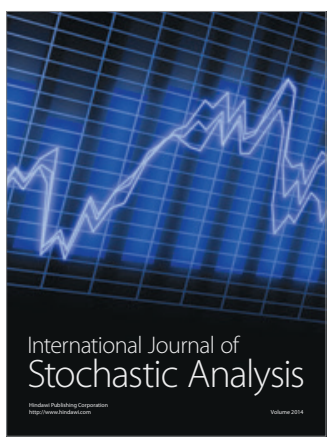

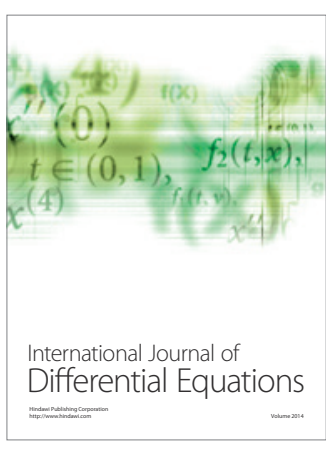
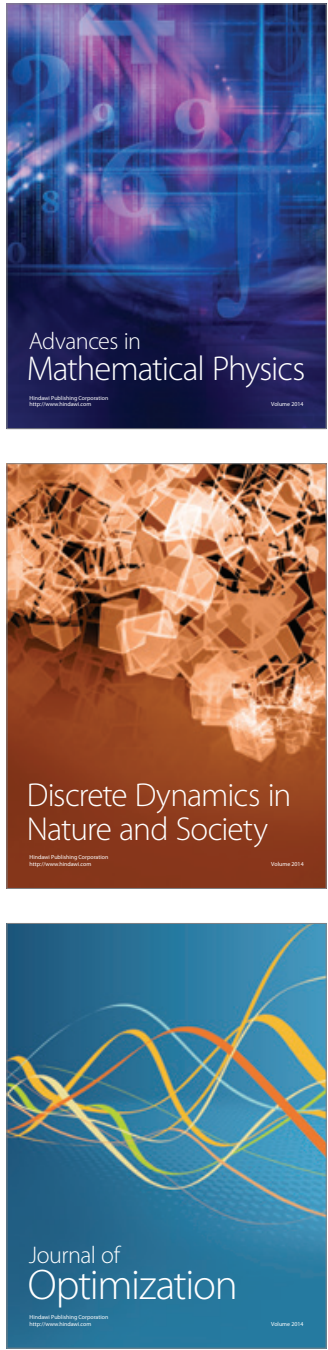